\title{
Effects of Unbalanced Polyunsaturated Fatty Acid Intake on Obesity-related Metabolic and Hepatic Dysfunctions
}

\author{
Giorgio Ricci ${ }^{*}$, Edgardo Canducci ${ }^{2}$, Ada Guida ${ }^{1}$, Rosario Arena ${ }^{1}$, Ambra Frascari ${ }^{1}$, Bruna Ravani², \\ Vittorio Alvisi ${ }^{3}$ \\ ${ }^{1}$ Postgraduate School of Gastroenterology, Section of Endocrinology and Internal Medicine, Department of \\ Medical Sciences, University of Ferrara, Ferrara, Italy; \\ ${ }^{2}$ Clinical Nutrition, Section of Internal \& Cardiopulmonary Medicine, Department of Medical Sciences, University \\ of Ferrara, Ferrara, Italy; \\ ${ }^{3}$ Internal Medicine Unit, Malatesta Novello Hospital, Via R. Serra 2, 47521 Cesena, Italy \\ Email: giorgio.ricci@unife.it
}

\begin{abstract}
In obesity, BARD (BMI, AST/ALT Ratio, presence of Diabetes) score, positive for values $\geqslant 2$, is a non-invasive marker of liver fibrosis. Unbalanced dietary habits are related to metabolic and liver dysfunctions. The aims were to investigate in Italian obese patients the prevalence of insulin resistance (IR) and positive BARD; the evaluation of excessive, or deficient, nutrient intakes and their relationship with IR and/or liver fibrosis. From the 220 records, 198 obese patients were included. Anthropometric measurements, HOMA-IR, BARD score and the nutrient intakes, as variances over or below the Recommended Dietary Allowances (RDAs) by the dietary assessment, were calculated as $50^{\text {th }}\left(25^{\text {th }}, 75^{\text {th }}\right)$ percentile. HOMA-IR was in $68 \%$ averaging $3.53(2.26,5.66)$, and positive BARD in $42 \%$. Excessive $\omega-6$ fatty acid intake was associated with high and significant risk (OR 5.01) of IR, as well as of liver fibrosis (OR 5.67). Excessive $\omega-6$ fatty acid intake enhances $\omega-6$ derived eicosanoids, inducing liver inflammation and fibrosis.
\end{abstract}

Keywords: Italian obese patients, dietary habits, omega-6 fatty acid, polyunsaturated fatty acid, liver fibrosis marker, insulin resistance

\section{Introduction}

Insulin resistance (IR) represents the main link between obesity, metabolic syndrome, and liver disease with fat accumulation, i.e. non-alcoholic fatty liver disease (NAFLD) [1]. In the majority of obese patients, NAFLD remains stable for many years. However, in a subset of these patients it may worsen to steatohepatitis (NASH) and liver cirrhosis [2]. The progression depends on the degree of liver fibrosis. The combination of anthropometric and serum markers could be useful to predict the severity of liver fibrosis in such patients [3]. A simple scoring system, i.e. BARD, is available and it uses four noninvasive, easily reproducible variables (BMI, AST/ALT Ratio, presence of Diabetes). Ranging from 2 to 4, the score is associated with an odds ratio of 17 to predict liver fibrosis, with a negative predicted value close to $100 \%[4,5]$. Although inadequate dietary habits in obesity are strongly related to the development of insulin resistance and liver dysfunction [6], few investigations have focused on the dietary habits in obese Italian subjects with the quantitative determination of nutrient intakes, and the relationships between dietary constituents and the development or progression of metabolic and liver disorders in obesity $[7,8]$. The aims of this study were to investigate the following aspects in an Italian group of obese patients: the prevalence of IR and positive BARD (score $\geqslant 2$ ); the nutrient intakes by quantitative determination; the evaluation of the excessive or deficient, i.e. unbalanced, intakes, calculating the rate of variance of each nutrient, as a percent value, over or below the Recommended Dietary Allowance (RDA) for the Italian population; the risk of IR and/or liver fibrosis in those cases with unbalanced nutrient intakes. 


\section{Methods}

A retrospective study was conducted on the records of obese patients (Body Mass Index, BMI $>30$ $\mathrm{kg} / \mathrm{m}^{2}$ ) who were admitted to our hospital department, from April 2010 to November 2014, for bariatric treatment of obesity. A total of 220 cases were obtained from the records, because they satisfied the main inclusion criteria, that were obesity, age range (20-70 yrs), and the presence of the required data (anthropometry, laboratory, dietary habit as quantitative nutritional assessment) in the records. A total of 22 cases were excluded for hepatitis B virus or hepatitis C virus positivity, alcohol consumption $>30$ $\mathrm{g} /$ day, use of medications with reported hepatosteatogenic effect (amiodarone, tamoxifen, estrogens), and type 1 diabetes.

BMI $\left(\mathrm{kg} / \mathrm{m}^{2}\right)$, waist circumference $(\mathrm{cm})$ and laboratory determinations were obtained from the records. Insulin resistance was calculated by the homeostasis model assessment (HOMA-IR) as fasting serum insulin $(\mu \mathrm{U} / \mathrm{mL}) \times$ fasting plasma glucose $(\mathrm{mmol} / \mathrm{L}) / 22.5$; values $>2.5$ indicate a state of IR [9]. The BARD score was calculated as previously reported [4]. Dietary habits were assessed with a standard 24$\mathrm{h}$ food recall and the detailed questioning by an experienced nutritionist determined the nutrient intakes using a computed database, according to the Italian table of food composition [10]. The descriptions of the portion size of each food (small, medium, large) and the evaluation of the portion size of the food using the photographic atlas of food portion size were used to calculate the amounts of nutrient intakes [11]. The RDAs for the Italian population were used as the reference range [12]. Moreover, in order to highlight the variation of each selected nutrient from the corresponding RDA, we calculated the rate of variance, as a percent value, over or below the RDA. Continuous variables are expressed as $50^{\text {th }}, 25^{\text {th }}$, $75^{\text {th }}$ percentiles; categorical variables are shown as number of patients and percentages. Statistical analyses were performed with IBM® SPSS ${ }^{\circledR} 22.0$ Statistics (SPSS, Chicago, IL, USA). For comparisons, the non-parametric Mann Whitney $U$ test was used; odds ratios (OR) of inadequate nutrient intakes $(95 \% \mathrm{CI})$, as risk factors for IR (HOMA $>2.5)$ and liver fibrosis (+ve BARD), were calculated by logistic regression. Statistical significance was defined as $\mathrm{p}<0.05$.

\section{$3 \quad$ Results}

The 198 selected cases (M/F 48/150) showed the following anthropometric and laboratory data: age 42 $(35,50)$ years, BMI $40(35,45) \mathrm{kg} / \mathrm{m}^{2}$, waist $117(105,128) \mathrm{cm}$, Impaired fasting glucose (IFG, glucose $100-125 \mathrm{mg} / \mathrm{dL}$ ) in $45 \%$ of the patients, Type 2 diabetes mellitus (T2DM) in 11\%, HOMA-IR in $68 \%$ averaging $3.53(2.26,5.66)$, and BARD positive (score $\geqslant 2)$ in $42 \%$. The daily intakes of selected nutrients are shown in Table 1 . The comparison with RDAs showed significant differences for most intakes. The variations, as percentages, of the nutrient intakes over or below the RDAs show that animal protein intakes averaged $273 \%$ over the RDA; fat intakes were $130 \%$ over RDA. The intake rates of total carbohydrates were $17 \%$ over the RDA. The simple carbohydrate intake averaged $68 \%$ over RDA. The $\omega-6$ fatty acid intake was $60 \%$ and the $\omega-6 / \omega-3$ fatty acid ratio was $101 \%$ over the RDA. Nutrients with protective effects, such as Polyunsaturated fatty acids (PUFA) and $\omega-3$ fatty acids, averaged $43 \%$ and $18 \%$ below RDAs, respectively. The fiber intake averaged $15 \%$ below the RDA. The unbalanced nutrient intakes are shown in the Table 1 as percentages of variations over or below the RDAs. The median values, as cutoff values, highlight the cases with excessive or deficient nutrient intakes. Their relationship to HOMA-IR and + ve BARD by logistic regression test shows those intakes associated with IR and/or +ve BARD. The excessive $\omega-6$ fatty acid intake is associated with a high and significant risk (OR 5.01) of IR, as well as of liver fibrosis (OR 5.67). The highly deficient PUFA intake is associated with a very elevated risk (OR 11.23) of IR and a less elevated risk (OR 3.88) of liver fibrosis. 
Table 1. Nutrient intakes in obese patients: comparison with recommended dietary allowances and the rate of variation versus recommended dietary allowances. The median values of variation, as the cut-off values of inadequate intake over or below the corresponding recommended dietary allowances, show their risk of association (Odds Ratio) with insulin resistance and liver fibrosis marker.

\begin{tabular}{l|c|c|c|c|c|c|c|c}
\hline \multicolumn{1}{c|}{ Nutrient intakes } & $\begin{array}{c}\text { Patients } \\
\mathbf{n}=\mathbf{1 9 8})\end{array}$ & $\mathbf{R D A}$ & \% variation & $\begin{array}{c}\text { Cutoff value } \\
\text { of inadequate } \\
\text { intake }\end{array}$ & $\begin{array}{c}\text { IR } \\
\text { Odds } \\
\text { Ratio }\end{array}$ & 95\%CI & $\begin{array}{c}\text { BARD } \\
\text { Odds } \\
\text { Ratio }\end{array}$ & 95\%CI \\
\hline Protein $(\mathrm{g})$ & $137(113,171)$ & $64(61,70)$ & $107(74,152)$ & $\geqslant 107$ & 0.74 & $0.31-1.76$ & 1.86 & $0.81-4.25$ \\
\hline Animal protein $(\mathrm{g})$ & $83(62,110)$ & $22(20,23)$ & $273(184,388)$ & $\geqslant 273$ & 1.15 & $0.48-2.76$ & 1.20 & $0.53-2.76$ \\
\hline Fat $(\mathrm{g})$ & $144(119,169)$ & $60(57,66)$ & $130(90,172)$ & $\geqslant 130$ & 1.20 & $0.52-2.77$ & 0.84 & $0.39-1.80$ \\
\hline SFA $(\mathrm{g})$ & $22(16,29)$ & $24(22,26)$ & $-9(-33,21)$ & $\geqslant-9$ & 1.28 & $0.52-3.14$ & 2.25 & $1.01-5.06$ \\
\hline MUFA $(\mathrm{g})$ & $52(43,62)$ & $48(46,53)$ & $5(-16,27)$ & $\leqslant 5$ & 1.44 & $0.62-3.34$ & 2.60 & $1.16-5.83$ \\
\hline PUFA $(\mathrm{g})$ & $10(8,13)$ & $17(16,19)$ & $-43(-58,-26)$ & $\leqslant-43$ & 11.23 & $2.09-60.30$ & 3.88 & $0.95-15.8$ \\
\hline$\omega-3$ fatty acid $(\mathrm{g})$ & $1(0.8,1.4)$ & $1.2(1.1,1.3)$ & $-18(-39,8.3)$ & $\leqslant-18$ & 1.13 & $0.46-2.78$ & 0.58 & $0.26-1.29$ \\
\hline$\omega-6$ fatty acid $(\mathrm{g})$ & $8(6.4,10.6)$ & $4.8(4.6,5.3)$ & $60(24,117)$ & $\geqslant 60$ & 5.01 & $0.94-27.40$ & 5.67 & $1.38-23.36$ \\
\hline$\omega-6 / \omega-3$ fatty acid ratio & $8(6.7,9.2)$ & 4 & $101(68,132)$ & $\geqslant 101$ & 0.46 & $0.20-1.04$ & 0.92 & $0.44-1.92$ \\
\hline Cholesterol $(\mathrm{mg})$ & $331(227,423)$ & 300 & $10(-24,40)$ & $\geqslant 10$ & 1.36 & $0.73-2.51$ & 2.01 & $1.09-3.68$ \\
\hline Total CHO $(\mathrm{g})$ & $442(373,517)$ & $364(346,407)$ & $17(-4.5,39)$ & $\geqslant 17$ & 1.21 & $0.61-2.40$ & 1.80 & $0.93-3.49$ \\
\hline Simple CHO $(\mathrm{g})$ & $119(88,164)$ & $69(66,76)$ & $68(23,137)$ & $\geqslant 68$ & 0.60 & $0.30-1.18$ & 0.83 & $0.43-1.62$ \\
\hline Fiber $(\mathrm{g})$ & $26(21,33)$ & 30 & $-15(-31,9)$ & $\leqslant-15$ & 1.42 & $0.74-2.74$ & 0.50 & $0.26-0.95$ \\
\hline
\end{tabular}

Legends: RDA: Recommended Dietary Allowance; IR: insulin resistance; CI: confidence interval; +ve BARD: positive score $(\geqslant 2)$ of liver fibrosis marker, BMI, AST/ALT Ratio, presence of Diabetes.

\section{Discussion}

Although dietary habits are strongly related to the development of obesity, few investigations adopted quantitative nutritional assessment to demonstrate the relationships between dietary constituents and the development of IR and liver fibrosis in NAFLD Italian obese patients. In agreement with previous reports [7,13], HOMA-IR was observed in the majority $(68 \%)$ of our obese patients. On the other hand, +ve BARD predicted liver fibrosis in $42 \%$ of our patients. Although all the intakes proved inadequate, some nutrient intakes showed a close relationship to the presence of metabolic and hepatic dysfunctions. The nutrient intakes were in most cases significantly higher than RDAs, and sometimes below for those nutrients with well-known protective effects, such as PUFA, especially $\omega-3$ fatty acids. We observed that the highest variation rates were not found for total carbohydrates, as could be expected, but for fat and protein, and especially for animal protein, averaging $273 \%$ over the RDA. As regards the intakes of those nutrients with protective effects, monounsaturated fatty acids (MUFA) were slightly over (5\%) the RDA; PUFA were significantly below the RDA (-43\%). The fiber intake was below (-15\%) the RDA. Moreover, the intake of $\omega-6$ fatty acids was $60 \%$ over the RDA. The current opinion is that hypercaloric foods with a high glycemic index induce IR and initialize the steps of liver damage $(6,14)$. However, not only carbohydrates, but all inadequate nutrient intakes may play an important role in the onset and progression of hepatic and metabolic disorders [7,15]. The carbohydrate energy intake could not be considered excessive because it only slightly (17\%) exceeded the RDA. The fat composition intake mainly consisted of monounsaturated fatty acids with a well-known protective effect, rather than saturated fatty acids. This finding may be related to the fact that the Italian diet is traditionally characterized by bread, pasta, and olive oil. Nevertheless, our study highlighted a considerable consumption of animal protein. This aspect clearly suggests a trend away from traditional Mediterranean eating towards Western dietary habits, in agreement with previous reports [8, 16-17]. Moreover, the $\omega-6$ fatty acid intake exceeded the RDA by $60 \%$. The excessive $\omega-6$ fatty acid intake would increase the levels of $\omega$-6-derived eicosanoids, that are potent mediators of thrombosis and inflammation, than $\omega$-3-derived eicosapentaenoic acid. The unbalanced $\omega-6 / \omega-3$ ratio would suggest the close relationship with progressive liver disease from steatosis towards inflammation and fibrosis [18-20]. 
Abbreviations. BMI, Body Mass Index; AST, aspartate aminotransferase; ALT, alanine aminotransferase; BARD, BMI, AST/ALT Ratio, presence of Diabetes; IR, insulin resistance; HOMA, homeostasis model assessment; RDA, recommended dietary allowance; $\omega-6,-3$, omega-6, -3; NAFLD, non-alcoholic fatty liver disease; NASH, non-alcoholic steatohepatitis; IFG, impaired fasting glucose; T2DM, Type 2 diabetes mellitus; PUFA, Polyunsaturated fatty acids; MUFA, monounsaturated fatty acids; SFA, saturated fatty acids; CHO, carbohydrates.

Acknowledgements. Funding of the present study was received from the "Academic Fund for the Scientific Research, University of Ferrara, 2012-2013"

\section{References}

1. A. Berlanga, E. Guiu-Jurado, J.A. Porras, and T. Augue, "Molecular pathways in non-alcoholic fatty liver disease". Clinical Experimental Gastroenterology; vol. 7, pp. 221-239, 2014.

2. P. Dietrich and C. Hellerbrand, "Non-alcoholic fatty liver disease, obesity and the metabolic syndrome" Best Practice \& Research Clinical Gastroenterology, vol. 28, no. 4, pp. 637-653, 2014.

3. C.F. Subasi, U.E. Aykut and Y. Yilmaz, "Comparison of noninvasive scores for the detection of advanced fibrosis in patients with nonalcoholic fatty liver disease" European Journal Gastroenterology \& Hepatology, vol. 27, no. 2, pp. 137-141, 2015.

4. S.A. Harrison, D. Oliver, H.L. Arnold, S Gogia and B.A. Neuschwander-Tetri, "Development and validation of a simple NAFLD clinical scoring system for identifying patients without advanced disease" Gut, vol.57, no.10, pp. 1441-1447, 2008.

5. N.C. Chavez-Tapia and C. Tiribelli, "Are non-invasive tests accurate enough to predict hepatic fibrosis in nonalcoholic fatty liver disease (NAFLD)?" Gut, vol. 57, no. 10, pp. 1351-1353, 2008.

6. E.M. McCarthy and M.E. Rinella, "The role of diet and nutrient composition in nonalcoholic fatty liver disease" Journal of Academy of Nutrition and Dietetics, vol. 112, no. 3, pp. 401-409, 2012.

7. G. Ricci, E. Canducci, V. Pasini, A. Rossi, G. Bersani, E. Ricci and V. Alvisi, "Nutrient intake in Italian obese patients: relationships with insulin resistance and markers of non-alcoholic fatty liver disease" Nutrition, vol. 27, no. 6, pp. 672-676, 2011.

8. G. Ricci, E. Canducci, A. Guida, A. Frascari, A. Rossi, G. Bersani, B. Ravani and V. Alvisi, "The gender-related differences of nutrient intakes in a group of Italian obese patients display the ongoing transition from Mediterranean to western dietary patterns" Obesity Surgery, vol. 24, no. 6 , pp. 965-967, 2014.

9. A. Borai, C. Livingstone, I. Kaddam and G. Ferns, "Selection of the appropriate method for the assessment of insulin resistance" BMC Medical Research Methodology, vol. 11, pp. 158, 2011.

10.E. Carnovale and L. Marletta, Tabelle di composizione degli alimenti. Ed EDRA, INRAN, Rome, Italy, 2000.

11.M. Nelson, M. Atkinson and S. Darbyshire, "Food photography II: use of food photographs for estimating portion size and the nutrient content of meals" British Journal of Nutrition, vol. 76, no. 1, pp. 31-49, 1996.

12.Società Italiana di Nutrizione Umana (S.I.N.U.). Livelli di Assunzione Raccomandati di Energia e Nutrienti per la Popolazione Italiana (LARN), IV revisione, SICS, Milan, Italy, 2014.

13.V. Ratziu, S. Bellentani, H. Cortez-Pinto, C. Day and G. Marchesini, "A position statement on NAFLD/NASH based on the EASL 2009 special conference" Journal of Hepatology, vol. 53, no. 2, pp. 372-384, 2010.

14.K.A. Lê and M. Bortolotti, "Role of dietary carbohydrates and macronutrients in the pathogenesis of nonalcoholic fatty liver disease" Current Opinion in Clinical Nutrition \& Metabolic Care, vol. 11, no. 4, pp. 477$482,2008$.

15.C. Gonzalez, V. de Ledinghen, J. Vergniol, J. Foucher, B. Le Bail, S. Carlier, E. Maury, H. Gin and V. Rigalleau "Hepatic steatosis, carbohydrate intake, and food quotient in patients with NAFLD" International Journal of Endocrinology, 428542. Published online, May 2, 2013.

16.E.M. Inelmen, E.D. Toffanello, G. Enzi, G. Sergi, A. Coin, L. Busetto and E. Manzato, "Differences in dietary patterns between older and younger obese and overweight outpatients" The journal of nutrition, health \& aging, vol. 12, no. 1, pp.3-8, 2008. 
17.L. Abenavoli, N. Milic, V. Peta, F. Alfieri, A. De Lorenzo and S. Bellentani, "Alimentary regimen in nonalcoholic fatty liver disease: Mediterranean diet" World Journal of Gastroenterology, vol. 20, no. 45, pp. 16831$16840,2014$.

18.B. Elisha, F. Guebre-Egziabher, H Vidal, J.P. Bastard, M. Laville and R. Rabasa-Lhoret, "From French to Mediterranean diet: importance of the omega-6/omega-3 fatty acids ratio" World Review of Nutrition and Dietetics, vol. 102, pp. 81-91, 2011.

19.J. Monteiro, M. Leslie, M.H. Moghadasian, B.M. Arendt, J.P. Allard, D.W. Ma, "The role of $n-6$ and $n-3$ polyunsaturated fatty acids in the manifestation of the metabolic syndrome in cardiovascular disease and nonalcoholic fatty liver disease" Food \& Function, vol. 5, pp. 426-435, 2014.

20.A.P. Simopoulous, "An increase in the Omega-6/Omega-3 fatty acid ratio increases the risk of obesity" Nutrients, vol. 8 , no. 3, pp. $128,2016$. 